\title{
Transmittance of tapered photonic crystal fibers with absorbing coatings
}

\author{
Mauricio Salazar Sicacha ${ }^{1}$, Vladimir P. Minkovich ${ }^{1, *}$, Alexander B. Sotsky ${ }^{2}$, Artur V. Shilov ${ }^{2}$, and Luidmila I. Sotskaya ${ }^{3}$ \\ ${ }^{1}$ Centro de Investigaciones en Optica, A.C., 37150, Mexico \\ ${ }^{2}$ A.A. Kuleshov Mogilev State University, 212009, Mogilev, Belarus \\ ${ }^{3}$ Belarussian-Russian University, 212000, Mogilev, Belarus
}

\begin{abstract}
The interaction of the adiabatically tapered photonic crystal fiber fundamental mode with a thinfilm absorbing coating, deposited on a surface of a taper waist, on transmission of a tapered fiber is studied. Examples of using this interaction in refractive index sensors and for detection of an adsorption layer with ammonia molecules upon contact of the absorbing coating with a liquid medium are presented. It is obtained that a pronounced sensory effect occurs in the case of a resonant coupling between the fundamental fiber mode and cladding modes localized between photonic crystal fiber air channels and the absorbing coating.
\end{abstract}

It is known that the fundamental mode of an index guiding photonic-crystal fiber (PCF) can be resonance coupled with leaky modes of a thin film PCF coating (cover) [1]. If the coating is absorbing, the coupling leads to a quasiperiodic spectral transmittance of the PCF that can be modified by interaction of coating modes with environment. However, at using the untapered PCF's mentioned mode, the coupling is rather weak. As a result, it can be only observed when sufficiently long stretches (tens of centimeters) of coated PCFs are used. Besides, to realize corresponding sensory effects it is necessary to keep a coating thickness $d$ constant along the entire sensitive stretch of the PCF with a tolerance of $0.075 \lambda / \sqrt{n_{c}^{2}-n_{s}^{2}}$, where $\lambda$ is the light wavelength, $n_{c}$ and $n_{s}$ are refractive indexes of the coating and a fiber cladding, respectively [1]. For long PCF stretches this requirement is difficult to fulfil. It is hoped that the use of adiabatic PCF tapers with a length of a sensitive region of a centimeter order will overcome these difficulties. Due to a small transverse size of a taper waist, the interaction of the fundamental PCF mode with an absorbing coating of the taper waist has some attractive features and therefore are considered in this report. Corresponding estimates are presented for transmission spectra $T(\lambda)$ of PCF tapers at sensing the refractive index of a liquid, and for detection of an adsorption molecular layer. Numerical simulations are performed with the use of the exact method of integral equations [1].

Fig. 1 illustrates a resonance mode coupling in an adiabatic PCF taper used as a liquid refractive index sensor. In modelling we used a home-made silica glass PCF with 3 hexagonal rings of air holes in its cross section. In our untapered PCF, cladding diameter $D_{0}=123.4 \mu \mathrm{m}$, diameter of air holes $a_{0}=3 \mu \mathrm{m}$, photonic crystal fiber pitch $\Lambda_{0}=8.8 \mu \mathrm{m}$ [2]. At the adiabatic taper fabrication, the diameter of holes $a$ and the pitch $\Lambda$ within the taper waist modify by the rule [3] $a=\left(D / D_{0}\right) a_{0}$, $\Lambda=\left(D / D_{0}\right) \Lambda_{0}$, where $D=44 \mu \mathrm{m}$ is the taper waist diameter; the length of the waist is $3 \mathrm{~cm} \mathrm{[2].}$

Fig. 1 refers to the waist coated with the absorbing coating of a multifunctional butyl acrylate with a thickness $d=5 \mu \mathrm{m}$ and the refractive index $n_{c}=1.54$ i0.00002. The left and right transmittance minima in Fig.1 correspond to a coupling of the fundamental PCF mode with TM and TE leaky modes of the coating, respectively, that realizes at the phase matching of the modes [1]. This coupling leads to a resonance energy capture of the fundamental PCF mode by the absorbing coating and a release of heat in it.

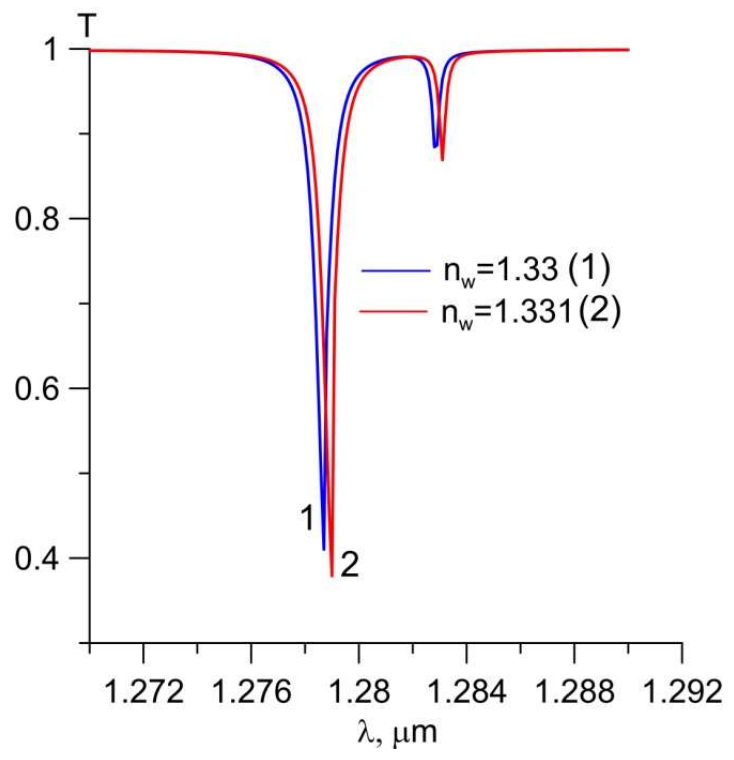

Fig.1. Shift of the taper transmittance spectrum with a change in the refractive index of a surrounding liquid.

$\overline{{ }^{*} \text { Corresponding author: vladimir@,cio.mx }}$ 
The sensory effect is explained by increase in effective mode indices of cover modes with increase in the refractive index of a liquid contacted with the absorbing coating.

Fig. 2 shows a modification of the taper transmission spectrum caused by formation of an adsorption layer of ammonia molecules with a thickness of $0.0085 \mu \mathrm{m}$ on a surface of the absorbing coating which consists of a tin dioxide doped with antimony (7\%) contacting with an aqueous ammonia. In this case $d=0.1 \mu \mathrm{m}, n_{c}=1.9082$ i0.00042. Due to a small $d$, the absorbing coating

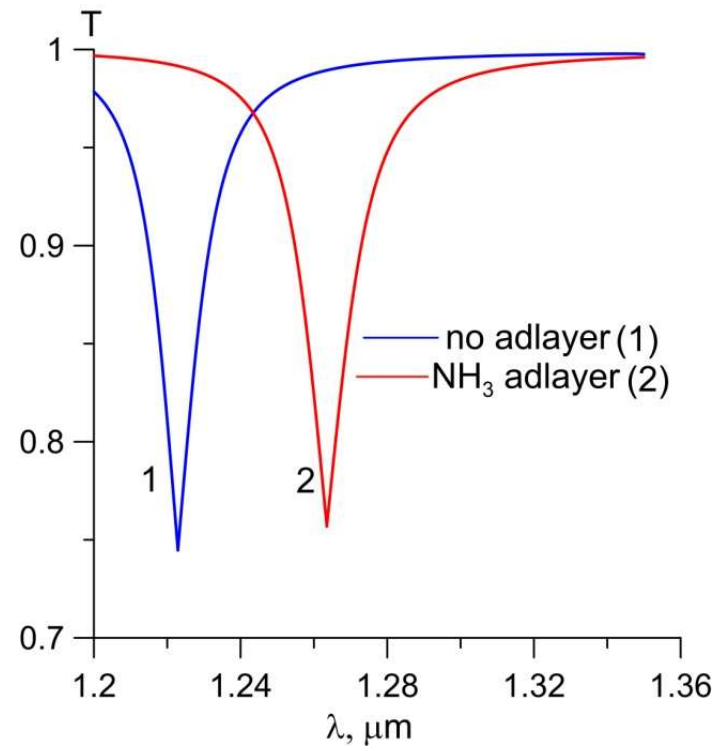

Fig.2. Shift of the PCF taper transmission spectrum at a formation of an adsorption layer of ammonia molecules on a surface of a tin dioxide coating.

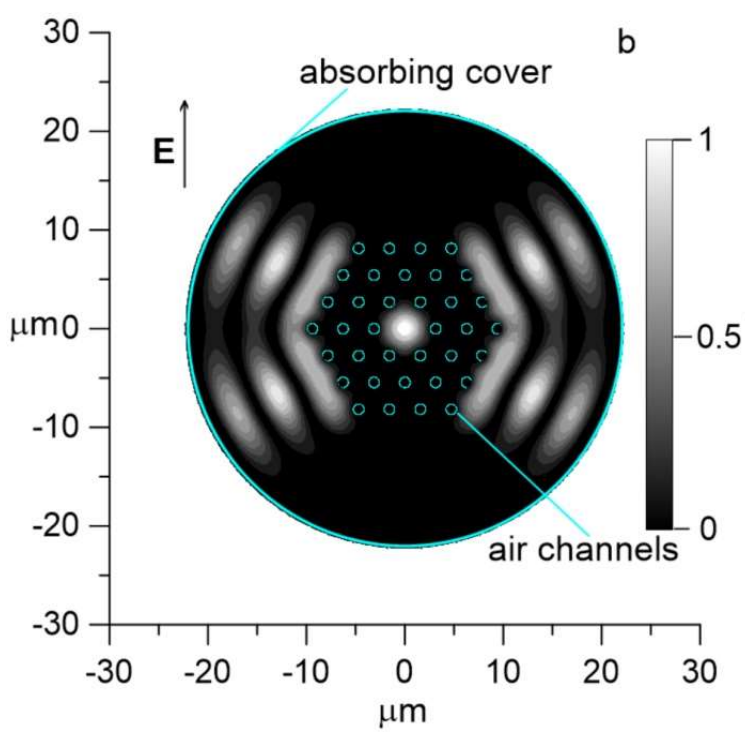

Fig.3. The optical density of the taper waist working mode at a minimal taper transmission in the case of the curve 1 in Fig. 2. The arrow $\mathbf{E}$ indicates the main orientation of a mode electric field vector. does not have leaky modes that can be in phase synchronism with the fundamental PCF mode in the spectral range of Fig. 2. In this case a sensory effect is explained by a resonance coupling of the fundamental PCF mode concentrated in the fiber core with a mode of the fiber cladding that concentrated in the area between air channels and coating [3]. This coupling mechanism is confirmed by Fig. 3 that illustrates a working mode intensity distribution at the wavelength corresponding to the transmission minimum for the curve 1 in Fig. 2. Cladding modes of the tapered PCF interact with the absorbing coating much more effective than the fundamental mode. This explains more pronounced sensory effect in Fig. 2 in comparison with Fig. 1. Calculations show, that necessary conditions for its realizations are high refractive index of the cover (coating) and rather small diameter of the taper waist. Only under these conditions and the mentioned mode coupling, the expressed standing waves are realized in the PCF cladding (see Fig. 3).

This work was partially supported by the State Scientific Research Program "Photonics, Opto- and Microelectronics 1.3.03" of the Belarus Republic.

\section{References}

1. A.B. Sotsky, L.I. Sotskaya, V.P. Minkovich, D. Monzon-Hernandez, Tech. Phys., 54, 6 (2009)

2. G.A. Cardenas-Sevilla, D. Monzon-Hernandez, V. P. Minkovich, Appl. Phys. Express, 5, 022502 (2012)

3. V.P. Minkovich, D. Monzon-Hernandez, J. Villatoro, A.B. Sotsky, L.I. Sotskaya, J. Lightw. Technol., 24, $11,(2006)$ 